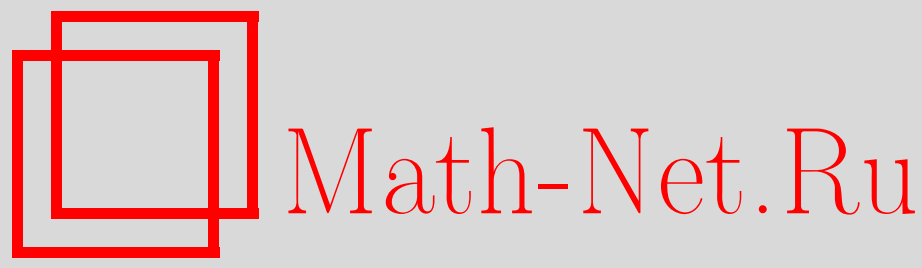

Н. В. Котляров, О существовании сколь угодно длинных слов, не содержащих квадратов с одной возможной ошибкой замещения, Дискрет. матем., 2015, том 27, выпуск 2, 56-72

DOI: https://doi.org/10.4213/dm1325

Использование Общероссийского математического портала Math-Net.Ru подразумевает, что вы прочитали и согласны с пользовательским соглашением http://www . mathnet.ru/rus/agreement

Параметры загрузки:

IP: 54.80 .97 .219

26 апреля 2023 г., 04:51:45

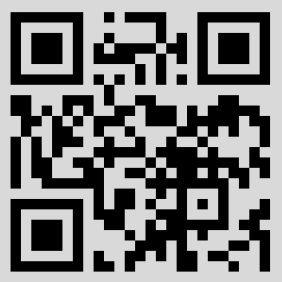




\title{
О существовании сколь угодно длинных слов, не содержащих квадратов с одной возможной ошибкой замещения
}

\author{
() 2015 г. Н. В. Котляров*
}

\begin{abstract}
Данная статья посвящена вопросам, связанным с существованием периодических структур в словах из формальных языков. Рассматриваются квадраты, то есть фрагменты вида $x x$, где $x$ - произвольное слово, и квадраты с одной ошибкой, то есть фрагменты вида $x y$, где слово $x$ отличается от слова $y$ ровно на одну букву. В данной статье изучаются условия существования сколь угодно длинных слов, не содержащих квадратов с длиной больше $l_{0}$ и квадратов $\mathrm{c}$ одной ошибкой и длиной больше $l_{1}$ в зависимости от натуральных чисел $l_{0}$ и $l_{1}$. Для всех возможных пар $l_{1} \geqslant l_{0}$ найден минимальный алфавит, над которым можно построить такое слово.

Работа выполнена при поддержке РФФИ, проект N 14-01-00598 (Вопросы синтеза, сложности и контроля управляющих систем), и программы фундаментальных исследований ОМН РАН “Алгебраические и комбинаторные методы математической кибернетики и информационные системы нового поколения" (проект "Задачи оптимального синтеза управляющих систем"). Также поддержка была оказана ИППИ РАН за счет гранта Российского научного фонда (проект № 14-50-00150).
\end{abstract}

Ключевые слова: последовательность Туэ, бесквадратные слова, словарная комбинаторика, ошибки замещения.

\section{1. Введение}

Данная статья посвящена вопросам, связанным с существованием периодических структур в словах из формальных языков. Наиболее простой и хорошо изученной периодической структурой являются квадраты, то есть фрагменты вида $x x$, где $x$ - произвольное непустое слово. Слово, не содержащее квадратов, называется бесквадратным. Классическим результатом, связанным с квадратами, является работа Акселя Туэ [1], в которой установлено существование как угодно длинных бесквадратных слов над алфавитом из трех букв. С другой стороны, несложно проверить, что не существует бесквадратных слов над алфавитом из двух букв. Поэтому из результатов Туэ следует, что алфавит из трех букв является минимальным алфавитом, над которым существуют как угодно длинные бесквадратные слова. В дальнейшем было получено много различных альтернативных доказательств данного

${ }^{*}$ Место работы: МГУ им. М. В. Ломоносова, Институт проблем передачи информации им. А.А. Харкевича РАН, e-mail: nikita.kotlyarov@bk.ru 
результата Туэ, одно из наиболее изящных доказательств представлено в [2]. Естественным обобщением результата Туэ является работа [3]. Рассматривались случаи, когда в словах допускаются достаточно "маленькие" квадраты [4] (в работе доказано существование бесконечного слова над алфавитом из двух букв, которое содержит только 3 различных квадрата). Другие базовые результаты, касающиеся квадратов, получены в работах $[5,6]$.

Нетрудно заметить, что задача существования сколь угодно длинных слов, не содержащих фрагментов определенного типа, эквивалентна задаче существования бесконечных слов над тем же алфавитом, не содержащих данных фрагментов. Поэтому в работах, посвященных этой тематике, обычно рассматривается эквивалентная задача существования бесконечных слов.

Слово называется сильно бескубным, если оно не содержит фрагментов вида $x x a$, где $x$ - непустое слово, $a$ - первая буква слова $x$. Еще одним классическим результатом Акселя Туэ в данной области является работа [3], в которой было доказано существование сколь угодно длинных сильно бескубных слов над двухбуквенным алфавитом. В частности, в данной работе был приведен пример бесконечного сильно бескубного слова над двухбуквенным алфавитом. Это слово называется в литературе последовательностью Туэ (Туэ-Морса).

Другим естественным обобщением задачи о существовании сколь угодно длинных бесквадратных слов является рассмотрение в качестве "запретных" фрагментов не только квадратов, но и квадратов с одной ошибкой замещения, то есть фрагментов вида $x y$, где слово $x$ отличается от слова $y$ ровно одной буквой. По нашим сведениям данная задача еще не рассматривалась в научной литературе. Отметим, что любой фрагмент слова длины 2 является либо квадратом, либо квадратом с одной ошибкой замещения, поэтому для данной задачи естественно вводить ограничения снизу как на длины "запретных" квадратов, так и на длины "запретных" квадратов с одной ошибкой замещения. Таким образом, в данной работе рассматривается следующая задача: для произвольных ограничений снизу на длину "запретных" квадратов и длину “запретных" квадратов с одной ошибкой замещения определить минимальную мощность алфавита, над которым существует бесконечное слово, удовлетворяющее данным ограничениям “запретных" фрагментов. В нашей работе содержится полное решение поставленной задачи.

\section{2. Основные определения}

Пусть $\Theta=\left\{\alpha_{1}, \alpha_{2}, \ldots, \alpha_{S}\right\}$ - некоторый алфавит, элементы алфавита будем называть буквами. Словом над алфавитом $\Theta$ называется конечная последовательность $x=\alpha_{i_{1}} \alpha_{i_{2}} \ldots \alpha_{i_{n}}$, состоящая из букв алфавита $\Theta$ (при этом допускается слово из нуля букв, которое называется nустым словом). Множество всех слов над алфавитом $\Theta$ обозначается через $\Theta^{*}$. Букву $\alpha_{i_{k}}$ будем обозначать через $x[k]$. Число $n$ называется длиной слова $x$ и обозначается через $|x|$. Фактором слова $x$ будем называть произвольный фрагмент $\alpha_{i_{k}} \ldots \alpha_{i_{k+l}}$ слова $x$, где $k \geqslant 1, k+l \leqslant n$. Заметим, что данный фактор слова $x$ однозначно определяется парой чисел $k$ и $k+l$, поэтому будем обозначать его через $x[k, k+l]$. Отметим, что $x[k]$ является частным случаем фактора. Два фактора $u$ и $v$ некоторого слова будем называть эквивалентными, если они являются одним и тем же фрагментом этого слова (эквивалентность этих факторов будем обозначать через $u \sim v)$. Заметим, что каждый фактор $w \sim x[k, l+k]$ можно рассматривать как слово $y=\alpha_{i_{k}} \ldots \alpha_{i_{k+l}}$ (будем обозначать это через $y=w$ ). Если 
два фактора $u$ и $v$ равны одному и тому же слову, то также будем обозначать это через $u=v$. Пусть $u \sim x[i, j], v \sim x[k, l]$ - два фактора слова $x$. Будем говорить, что $u$ содержится в $v$, если $i \geqslant k, j \leqslant l$. Отметим, что в этом случае $u$ является фактором $v$, поэтому $u$ может быть обозначено через $v[i-k+1, l-j+1]$. Длина фактора $x[i, j]$ равна $j-i+1$. Будем говорить, что фактор $w=x[j, j+k]$ находится слева от фактора $u=x[i, i+l]$, если $j+k+1=i$. Аналогично вводится понятие «находится справа».

Определим сверхслово или бесконечное слово как счетную последовательность букв алфавита $\alpha_{i_{1}} \alpha_{i_{2}} \ldots$ Множество всех сверхслов над алфавитом $\Theta$ обозначается через $\Theta^{\omega}$. Аналогично понятию фактора конечного слова вводится понятие фактора бесконечного слова. Префиксом (конечного или бесконечного) слова $x$ будем называть фактор $x[1, l]$. Соответственно, суффиксом конечного слова $x$ будем называть фактор вида $x[l, n]$, где $n=|x|$. Будем называть квадратом фактор вида $u v$, где $u=v$, при этом $u$ и $v$ называются соответственно левым и правым корнями данного квадрата. Периодом квадрата $w$ называется число $|w| / 2$, равное длине его корней. Cтрогим фактором слова $x$ будем называть фактор, который не является суффиксом или префиксом $x$. Пусть $u$ и $v$ - два слова одинаковой длины, большей либо равной $k$. Будем говорить, что $u$ и $v$ отличаются $k$ буквами, если $u[i] \neq v[i]$ ровно для $k$ значений $i$. Квадратом с одной ошибкой будем называть фактор вида $u v$, где $u$ отличается одной буквой от $v$. Аналогично, $u$ и $v$ будем называть соответственно левым и правым корнями данного квадрата с одной ошибкой. Периодом квадрата $w$ с одной ошибкой называется число $|w| / 2$, равное длине его корней. Конечное или бесконечное слово $x$ называется силъно бескубным, если оно не содержит фактора вида $u и a$, где $u$ - некоторое слово и $u[1]=a$.

Пусть $x$ - некоторое слово длины $n$. Промежутком в слове $x$ будем называть целое число $k$ от 1 до $n-1$, задающее позицию между буквами $x[k], x[k+1]$. Пусть $u \sim x[i, j]$ - некоторый фактор четной длины в слове $x$. Серединой фактора $u$ будем называть промежуток $(i+j-1) / 2$ в слове $x$. В дальнейшем в записи фактора в виде конкатенации других факторов, слов или букв его середину будем обозначать формальным символом |, не являющемся буквой.

Пусть $\Theta$ - некоторый алфавит. Будем называть морфизмом отображение $h$ : $\Theta^{*} \rightarrow \Theta^{*}$, удовлетворяющее условию: $h(x y)=h(x) h(y)$. Пусть для некоторого $a \in \Theta$ образ $h(a)$ начинается с буквы $a$ и имеет длину больше 1 . Тогда очевидно, что $h^{i}(a)$ является префиксом $h^{j}(a)$ для любых $i<j$, где $h^{i}(a)$ есть композиция $i$ отображений $h$, примененных последовательно к $a$. Поэтому можно рассмотреть сверхслово, содержащее в качестве префиксов все слова $h^{i}(a), i=1,2,3 \ldots$ Будем обозначать это сверхслово через $\hat{h}(a)$. Рассмотрим такой морфизм $\chi:\{0,1\}^{*} \rightarrow$ $\{0,1\}^{*}$, что $\chi(0)=01, \chi(1)=10$. Сверхслово $\hat{\chi}(0)$ называется последовательностью Туэ-Морса. В дальнейшем эту последовательность будем обозначать через $\Omega$. В [3] показано, что $\Omega$ обладает свойством сильной бескубности.

Пусть $k_{0}, k_{1}$ - натуральные числа.

Сформулируем свойство $\left(\begin{array}{c}* \\ k_{0}\end{array}\right)$ для (сверх)слова $x$ следующим образом: “у (сверх)слова $x$ нет факторов, являющихся квадратами с периодом больше $k_{0}$ ".

Сформулируем свойство $\left(\begin{array}{l}* * \\ k_{1}\end{array}\right)$ для (сверх)слова $x$ следующим образом: “у (сверх)слова $x$ нет факторов, являющихся квадратами с одной ошибкой и периодом больше $k_{1} "$.

В данной работе мы будем рассматривать естественный случай сверхслов, обладающих свойствами $\left(\begin{array}{c}* \\ k_{0}\end{array}\right)$ и $\left(\begin{array}{c}* * \\ k_{1}\end{array}\right)$ для $k_{1} \geqslant k_{0}$. 
Имеет место следующие очевидные свойства:

Утверждение 1. Если некоторое сверхслово обладает свойствами $\left(\begin{array}{l}* \\ k_{0}\end{array}\right)$ u $\left(\begin{array}{l}* * \\ k_{1}\end{array}\right)$, то это сверхслово обладает также свойствами $\left(\begin{array}{c}* \\ k_{0}^{\prime}\end{array}\right) u\left(\begin{array}{c}* * \\ k_{1}^{\prime}\end{array}\right)$, для любых $k_{0}^{\prime} \geqslant k_{0}, k_{1}^{\prime} \geqslant k_{0}$.

Утверждение 2. Если для некоторых $k_{0}, k_{1}$ не существует сверхслова, обладающего свойствами $\left(\begin{array}{c}* \\ k_{0}\end{array}\right)$ u $\left(\begin{array}{c}* * \\ k_{1}\end{array}\right)$, то для $k_{0}^{\prime} \leqslant k_{0}, k_{1}^{\prime} \leqslant k_{0}$ не существует сверхслова, обладающего свойствами $\left(\begin{array}{c}* \\ k_{0}^{\prime}\end{array}\right)$ u $\left(\begin{array}{c}* * \\ k_{1}^{\prime}\end{array}\right)$.

Обозначим через $\sigma\left(k_{0}, k_{1}\right)$ минимальный размер алфавита, над которым можно построить сверхслово, обладающее одновременно свойствами $\left(\begin{array}{c}* \\ k_{0}\end{array}\right)$ и $\left(\begin{array}{l}* * \\ k_{1}\end{array}\right), k_{1} \geqslant k_{0}$. В данной статье мы находим значение $\sigma\left(k_{0}, k_{1}\right)$ для любых $k_{0}, k_{1}$.

Для этого мы будем строить сверхслова с требуемыми свойствами над заданным алфавитом $\Delta$ из $S$ букв следующим образом. Пусть $\Theta=\{0,1\}, g$ такое отображение из $\Theta^{2}$ в $\Delta^{*}$, что все образы $g(00), g(01), g(10)$ и $g(11)$ имеют одинаковую длину, которую будем называть длиной отображения $g$. Определим отображение $f_{g}$ из $\Theta^{*} \cup \Theta^{\omega}$ в $\Delta^{*} \cup \Delta^{\omega}$ следующим образом:

$$
\begin{gathered}
f_{g}\left(a_{1} a_{2} \ldots a_{n-1} a_{n}\right)=g\left(a_{1} a_{2}\right) g\left(a_{2} a_{3}\right) \ldots g\left(a_{n-1} a_{n}\right), \\
f_{g}\left(a_{1} a_{2} \ldots\right)=g\left(a_{1} a_{2}\right) g\left(a_{2} a_{3}\right) \ldots
\end{gathered}
$$

Отметим, что отображение $f_{g}$ определено только для слов длины больше 1. Слова $g(00), g(01), g(10)$ или $g(11)$ будем называть элементами отображения $g$. Через $\Psi_{g}$ будем обозначать сверхслово $f_{g}(\Omega)$. Все сверхслова с требуемыми свойствами будут строиться как сверхслова $\Psi_{g}$ для некоторого специально подобранного $g$.

Пусть $x$ - некоторое (сверх)слово над $\Theta, u \sim x[i, j]-$ фактор $x$. Тогда бактор-образом $f_{g}(u)$ фактора $u$ будем называть фактор $v \sim f_{g}(x[i, j])$ (сверх)слова $f_{g}(x)$. Пусть $v$ - некоторый фактор $f_{g}(x)$. Будем называть фактор $u$ в $x$ бактор-прообразом фактора $v$, если $v$ является фактором фактор-образа $f_{g}(u)$ и для любого $w$, являющегося фактором $u$, отличным от $u$, слово $v$ не является фактором фактор-образа $f_{g}(w)$. Будем называть факторы сверхслова $\Psi_{g}$, являющиеся фактор-образами факторов $\Omega$ длины 2 , монообразами. Если $\alpha \beta \tau$ является фактором $\Omega$, где $\alpha, \beta, \tau \in\{0,1\}$, то $f_{g}(\alpha \beta)$ и $f_{g}(\beta \tau)$ называются последовательными монообразами. Учитывая, что последовательность Туэ-Морса является сильно бескубной, получаем, что парами последовательных монообразов являются $f_{g}(00)$ и $f_{g}(01), f_{g}(01)$ и $f_{g}(10), f_{g}(01)$ и $f_{g}(11), f_{g}(10)$ и $f_{g}(00), f_{g}(10)$ и $f_{g}(01), f_{g}(11)$ и $f_{g}(10)$.

Пусть $u \sim \Psi_{g}[i, j]$ - некоторый фактор $\Psi_{g}$. Будем называть расширением фактора $u$ слово $x$ наименьшей длины, которое является образом некоторого слова $y$ при отображении $g$ и содержит $u$ в качестве такого фактора $x\left[i^{\prime}, j^{\prime}\right]$, что $i^{\prime} \equiv i(\bmod d)$. В этом случае слово $у$ будем называть прообразом фактора $u$. Очевидно, что для фактора $u$ имеется по крайней мере один прообраз, являющийся его фактор-прообразом. С другой стороны, несложно заметить, что фактор $u$ может иметь несколько различных прообразов. Будем называть фактор и уникальным, если он имеет единственный прообраз.

\section{3. Вспомогательные утверждения}

Докажем несколько вспомогательных утверждений. 
Лемма 1. Любой фактор длины 7 сверхслова $\Omega$ является одним из следующих 22 слов: 0100110, 0101100, 0110010, 0110100, 0011010, 0010110, 0100101, 0101101, 0110011, 0110101, 0011001, 1011010, 1010010, 1001100, 1001010, 1100110, 1011001, 1010011, 1001101, 1001011, 1100101, 1101001.

Доказательство. Слова, перечисленные в формулировке леммы, являются всеми сильно бескубными словами над алфавитом $\{0,1\}$ длины 7. Заметим, что так как сверхслово $\Omega$ является сильно бескубным, то любой фактор $\Omega$ является сильно бескубным. Следовательно, любой фактор $\Omega$ длины 7 является одним из 22 слов, перечисленных в формулировке леммы. Лемма доказана.

Лемма 2. Пусть $g: \Theta^{2} \rightarrow \Delta^{*}$ - отображение длины $d$ и все значения отображения $g$ различны. Тогда $\Psi_{g}$ не содержит уникальных квадратов с периодом, кратным $d$.

Доказательство. Предположим обратное. Пусть $\Psi_{g}$ содержит некоторый уникальный квадрат $w$ с периодом, кратным $d$. Заметим, что $w \sim u_{1} \gamma_{1} \ldots \gamma_{n} u_{2} \mid v_{1} \delta_{1} \ldots \delta_{n} v_{2}$, где $\gamma_{1}, \ldots, \gamma_{n}, \delta_{1}, \ldots, \delta_{n}-$ монообразы, и $\left|u_{1}\right|=\left|v_{1}\right|<d,\left|v_{2}\right|=\left|u_{2}\right|<d$. Согласно определению квадрата имеем $u_{1}=v_{1}, u_{2}=v_{2}, \gamma_{i}=\delta_{i}, i=1,2, \ldots, n$.

Предположим, что $\left|u_{1}\right|>0$. Обозначим через $q$ фактор-прообраз $w$. Заметим, что $|q|=2 n+4$ и

1) $u_{1}$ является суффиксом $g(q[1] q[2])$,

2) $g(q[i+1] q[i+2])=\gamma_{i}=\delta_{i}=g(q[n+2+i] q[n+3+i])$ для $i=1, \ldots, n$,

3) $g(q[n+2] q[n+3])=u_{2} v_{1}$,

4) $v_{2}$ является префиксом $g(q[2 n+3] q[2 n+4])$.

Из п. 2 в силу того, что все элементы $g$ различны, получаем, что $q[i+1]=q[n+2+i]$ для $i=1, \ldots, n+1$. Обозначим через $q^{\prime}$ слово, которое получается из $q$ при замене $q[1]$ на $q[n+2]$, а $q[2 n+4]$ на $q[n+3]$. Из того, что $u_{1}=v_{1}$ и $u_{2}=v_{2}$, вытекает, что $u_{1}$ является суффиксом $g(q[n+2] q[n+3])$, аналогично, $v_{2}$ является префиксом $g(q[n+2] q[n+3])$. Поэтому, как легко заметить, $q^{\prime}$ является прообразом $w$. Поскольку $w$ является уникальным, получаем, что $q=q^{\prime}$, поэтому $q[1]=q[n+2], q[n+3]=$ $q[2 n+4]$. Следовательно, $q[1]=q[n+2]=q[2 n+3]$ и $q[1, n+1]=q[n+2,2 n+2]$, откуда получаем противоречие с сильной бескубностью $\Omega$.

Рассмотрим теперь случай $\left|u_{1}\right|=0$. Заметим, что в этом случае $\left|u_{2}\right|=0$. Обозначим через $q$ фактор-прообраз $w$. Заметим, что $|q|=2 n+1$ и $g(q[i] q[i+1])=\gamma_{i}=$ $\delta_{i}=g(q[n+i] q[n+1+i])$ для $i=1, \ldots, n$. Следовательно, поскольку все элементы отображения $g$ различны, получаем, что $q[i]=q[n+i], q[i+1]=q[n+i+1]$ для $i=1, \ldots, n$. Отсюда вытекает, что $q[1]=q[n+1]=q[2 n+1]$ и $q[2, n]=q[n+2,2 n]$, откуда получаем противоречие с сильной бескубностью $\Omega$. Лемма доказана.

\section{4. Двухбуквенный алфавит}

Теорема 1. Существует бесконечное слово над алфавитом из двух букв, которое обладает свойствами $\left(\begin{array}{c}* \\ 3\end{array}\right)$ u $\left(\begin{array}{c}* * \\ 3\end{array}\right)$.

Доказательство. Рассмотрим отображение $g_{1}: 00 \mapsto 010010,01 \mapsto 111000,10 \mapsto$ 000111, $11 \mapsto 101101$. Покажем, что для сверхслово $\Psi_{g_{1}}$ обладает свойствами $\left(\begin{array}{l}* \\ 3\end{array}\right)$ и $\left(\begin{array}{c}* * \\ 3\end{array}\right)$.

Сначала убедимся, что $\Psi_{g_{1}}$ не содержит в качестве факторов квадратов или квадратов с одной ошибкой с таким периодом $p$, что $3<p \leqslant 16$. Заметим, что длина 
фактор-прообразов таких факторов не превосходит 7. Поэтому нам достаточно проверить, что в $\Omega$ фактор-образ любого фактора длины 7 при отображении $f_{g_{1}}$ не содержит таких факторов. Согласно лемме 1 любой фактор длины 7 в $\Omega$ является одним из 22 слов, перечисленных в утверждении леммы. Таким образом, используя компьютерные вычисления, достаточно проверить, что при отображении $f_{g_{1}}$ образ любого из этих 22 слов не содержит таких квадратов или квадратов с одной ошибкой.

Теперь докажем, что $\Psi_{g_{1}}$ не содержит факторов, являющихся квадратами с периодом больше 16. Предположим обратное: пусть $\Psi_{g_{1}}$ содержит квадрат $w$ с периодом, большим 16. Заметим, что в $\Psi_{g_{1}}$ любой фактор длины 16 содержит в качестве фактора монообраз, поэтому левый корень этого квадрата содержит некоторый фактор $\Psi_{g_{1}}[i, i+5]$, являющийся монообразом. Отметим, что $\Psi_{g_{1}}[i, i+5]=\Psi_{g_{1}}[i+p, i+p+5]$. Предположим, что $\Psi_{g_{1}}[i+p, i+p+5]$ не является монообразом. Тогда $\Psi_{g_{1}}[i+p, i+p+5]$ содержится в качестве строгого фактора в конкатенации двух последовательных монообразов в $\Psi_{g_{1}}$. Тем самым получаем противоречие с тем, что, как несложно проверить, ни один элемент отображения $g_{1}$ не является строгим фактором конкатенации двух последовательных монообразов этого отображения. Таким образом, $\Psi_{g_{1}}[i+p, i+p+5]$ является монообразом. Следовательно, период квадрата $w$ кратен длине отображения $g$, т.е. $w \sim u_{1} \gamma_{1} \ldots \gamma_{k} u_{2} \mid v_{1} \delta_{1} \ldots \delta_{k} v_{2}$, где $k \geqslant 2$ и $\gamma_{1}, \ldots, \gamma_{k}, \delta_{1}, \ldots, \delta_{k}$ - такие монообразы, что $\gamma_{i}=\delta_{i}, i=1,2, \ldots, k$, и $0 \leqslant\left|u_{1}\right|,\left|u_{2}\right|<6, u_{1}=v_{1}, u_{2}=v_{2}$.

Пусть $\left|u_{1}\right|=0$, т.е. $w \sim \gamma_{1} \ldots \gamma_{k} \mid \delta_{1} \ldots \delta_{k}$. Тогда, поскольку все элементы $g_{1}$ различны, квадрат $w$ является уникальным, что противоречит лемме 2. Теперь рассмотрим случай $\left|u_{1}\right|>0$, т.е. $w \sim u_{1} \gamma_{1} \ldots \gamma_{k} u_{2} \mid v_{1} \delta_{1} \ldots \delta_{k} v_{2}$, где $0<\left|u_{1}\right|,\left|u_{2}\right|<6$. Заметим, что в этом случае $u_{2} v_{1}$ является монообразом, тем самым $\left|u_{2}\right|+\left|v_{1}\right|=\left|u_{1}\right|+\left|v_{2}\right|=6$. Рассмотрим произвольный прообраз $q$ квадрата $w$. Заметим, что $q$ имеет длину $2 k+4$, при этом $\gamma_{i}=g_{1}(q[i+1] q[i+2]), \delta_{i}=g_{1}(q[i+k+2] q[i+k+3])$ для $i=1,2, \ldots, k$. Таким образом, поскольку все элементы $g_{1}$ различны, буквы $q[2], q[3], \ldots, q[2 k+3]$ прообраза $q$ однозначно определяются квадратом $w$. Кроме того, из того, что $\gamma_{i}=\delta_{i}$, следует, что $q[2, k+2]=q[k+3,2 k+3]$. Заметим также, что $u_{1}$ является суффиксом в $g_{1}(q[1] q[2])$, а $v_{2}$ является префиксом в $g_{1}(q[2 k+3] q[2 k+4])$. Рассмотрим отдельно следующие подслучаи.

(1) Пусть $\left|u_{1}\right|,\left|v_{2}\right| \geqslant 2$. Заметим, что все элементы $g_{1}$ имеют как различные префиксы длины 2, так и различные суффиксы длины 2. Поэтому в этом случае буква $q[1]$ однозначно определяется из $u_{1}$, а буква $q[2 k+4]$ однозначно определяется из $v_{2}$. Таким образом, прообраз $q$ однозначно определяется квадратом $w$, т.е. $w$ является уникальным, что противоречит лемме 2.

(2) Пусть $\left|u_{1}\right|=1$. Заметим, что в этом случае $\left|v_{2}\right|=5$, тем самым аналогично подслучаю 1$)$ буква $q[2 k+4]$ однозначно определяется из $v_{2}$. Отметим также, что для любой буквы $a=0,1$ элементы $g_{1}(0 a)$ и $g_{1}(1 a)$ заканчиваются разными буквами. Поэтому буква $q[1]$ однозначно определяется, исходя из $u_{1}$ и буквы $q[2]$. Таким образом, и в этом случае квадрат $w$ является уникальным, что противоречит лемме 2.

(3) Случай $\left|v_{2}\right|=1$. Заметим, что в этом случае $\left|u_{1}\right|=5$, тем самым аналогично подслучаю 1) буква $q[1]$ однозначно определяется из $u_{1}$. Отметим также, что для любой буквы $a=0,1$ элементы $g_{1}(a 0)$ и $g_{1}(a 1)$ начинаются с разных букв. Поэтому буква $q[2 n+4]$ однозначно определяется, исходя из $v_{2}$ и буквы $q[2 n+3]$. Таким образом, и в этом случае квадрат $w$ является уникальным, что противоречит лемме 2 . 
Таким образом, для $\Psi_{g_{1}}$ мы доказали свойство $\left(\begin{array}{l}* \\ 3\end{array}\right)$.

Теперь докажем, что $\Psi_{g_{1}}$ не содержит факторов, являющихся квадратами с одной ошибкой и периодом, большим 16. Предположим, что $\Psi_{g_{1}}$ содержит квадрат $w$ с одной ошибкой и с периодом $p$, большим 16 . Заметим, что в $\Psi_{g_{1}}$ любой фактор длины больше 16 содержит в качестве фактора 2 смежных монообраза, поэтому левый корень квадрата $w$ содержит некоторые факторы $\Psi_{g_{1}}[i, i+5]$ и $\Psi_{g_{1}}[i+6, i+11]$, являющиеся монообразами. Так как корни квадрата отличаются одной буквой, то либо $\Psi_{g_{1}}[i, i+5]=\Psi_{g_{1}}[i+p, i+p+5]$, либо $\Psi_{g_{1}}[i+6, i+11]=\Psi_{g_{1}}[i+p+6, i+p+11]$. Поэтому, аналогично доказательству свойства $\left(\begin{array}{l}* \\ 3\end{array}\right), \Psi_{g_{1}}[i+p, i+p+5]$ и $\Psi_{g_{1}}[i+p+$ $6, i+p+11]$ являются монообразами. Следовательно, период квадрата $w$ кратен длине отображения $g$, т.е. $w \sim u_{1} \gamma_{1} \ldots \gamma_{k} u_{2} \mid v_{1} \delta_{1} \ldots \delta_{k} v_{2}$, где $k \geqslant 2$ и $\gamma_{1}, \ldots, \gamma_{k}, \delta_{1}, \ldots, \delta_{k}$ - монообразы, $i=1,2, \ldots, k$, и $0 \leqslant\left|u_{1}\right|,\left|u_{2}\right|<6,\left|u_{1}\right|=\left|v_{1}\right|,\left|u_{2}\right|=\left|v_{2}\right|$.

Пусть $\left|u_{1}\right|=0$, то есть квадрат $w$ с ошибкой имеет вид $\gamma_{1} \ldots \gamma_{k} \mid \delta_{1} \ldots \delta_{k}$, где $\gamma_{1}, \ldots, \gamma_{k}, \delta_{1}, \ldots, \delta_{k}$ являются монообразами, $k \geqslant 2$. Следовательно, квадрат $w$ с ошибкой содержит 2 монообраза $\gamma_{i}$ и $\delta_{i}$, которые отличаются друг от друга ровно одной буквой. Несложно проверить, что любые 2 элемента отображения $g_{1}$ либо совпадают, либо отличаются более чем одной буквой. Следовательно, такой случай невозможен. Теперь рассмотрим случай $\left|u_{1}\right|>0$, т.е. $w \sim u_{1} \gamma_{1} \ldots \gamma_{k} u_{2} \mid v_{1} \delta_{1} \ldots \delta_{k} v_{2}$, где $0<\left|u_{1}\right|,\left|u_{2}\right|<6$. Поскольку любые два элемента отображения $g_{1}$ либо совпадают, либо отличаются более чем одной буквой, имеем $\gamma_{i}=\delta_{i}, i=1, . ., k$. Поэтому либо $u_{1}$ отличается одной буквой от $v_{1}$, либо $u_{2}$ отличается одной буквой от $v_{2}$. Заметим, что в этом случае $u_{2} v_{1}$ является монообразом, тем самым $\left|u_{2}\right|+\left|v_{1}\right|=\left|u_{1}\right|+\left|v_{2}\right|=6$. Рассмотрим произвольный прообраз $q$ квадрата $w$. Заметим, что $q$ имеет длину $2 k+4$, при этом $\gamma_{i}=g_{1}(q[i+1] q[i+2]), \delta_{i}=g_{1}(q[i+k+2] q[i+k+3])$ для $i=1,2, \ldots, k$. Таким образом, поскольку все элементы $g_{1}$ различны, буквы $q[2], q[3], \ldots, q[2 k+3]$ прообраза $q$ однозначно определяются квадратом $w$ и $q[2, k+2]=q[k+3,2 k+3]$. Заметим также, что $u_{1}$ является суффиксом в $g_{1}(q[1] q[2])$, а $v_{2}$ является префиксом в $g_{1}(q[2 k+3] q[2 k+4])$. Рассмотрим отдельно следующие подслучаи.

(1) Пусть $\left|u_{1}\right|,\left|v_{2}\right| \geqslant 2$. Заметим, что все элементы $g_{1}$ имеют как различные префиксы длины 2, так и различные суффиксы длины 2. Поэтому в этом случае буква $q[1]$ однозначно определяется из $u_{1}$, а буква $q[2 k+4]$ однозначно определяется из $v_{2}$. При этом, если $u_{1}$ отличается одной буквой от $v_{1}$, то $u_{2}=v_{2}$, и, следовательно, $q_{k+2} q_{k+3}=q_{2 k+3} q_{2 k+4}$. В этом случае $q[2] \ldots q[k+2]=q[k+3] \ldots q[2 k+3], q[2]=q[k+3]=q[2 k+4]$, что противоречит сильной бескубности $\Omega$. Если $u_{2}$ отличается одной буквой от $v_{2}$, то $u_{1}=v_{1}$, и, следовательно, $q_{1} q_{2}=q_{k+2} q_{k+3}$. В этом случае $q[2] \ldots q[k+2]=q[k+3] \ldots q[2 k+3]$, $q[1]=q[k+2]=q[2 k+3]$, что противоречит сильной бескубности $\Omega$.

(2) Пусть $\left|u_{1}\right|=1$. Тогда $\left|v_{2}\right|=5$. Отметим, что префиксы длины 5 любых двух различных элементов отображения отличаются, как минимум, двумя буквами. Значит, $u_{2}=v_{2}$, следовательно, $q_{k+2} q_{k+3}=q_{2 k+3} q_{2 k+4}$. В этом случае $q[2] \ldots q[k+2]=q[k+3] \ldots q[2 k+3], q[2]=q[k+3]=q[2 k+4]$, что противоречит сильной бескубности $\Omega$.

(3) Пусть $\left|v_{2}\right|=1$. Тогда $\left|u_{1}\right|=5$. Этот случай рассматривается аналогично подслучаю 2), так как суффиксы длины 5 любых двух различных элементов отображения $g_{1}$ отличаются, как минимум, на две буквы.

Таким образом, для $\Psi_{g_{1}}$ мы доказали свойство $\left(\begin{array}{c}* * \\ 3\end{array}\right)$. Теорема доказана.

Теорема 2. Существует бесконечное слово над алфавитом из 2 букв, которое обладает свойствами $\left(\begin{array}{c}* \\ 2\end{array}\right)$ и $\left(\begin{array}{c}* * \\ 4\end{array}\right)$. 
Доказательство. Рассмотрим отображение $g_{2}: 00 \mapsto 000001,01 \mapsto 010100,10 \mapsto$ 011111, $11 \mapsto 011001$. Покажем, что сверхслово $\Psi_{g_{2}}$ обладает свойствами $\left(\begin{array}{c}* \\ 2\end{array}\right)$ и $\left(\begin{array}{c}* * \\ 4\end{array}\right)$.

Сначала убедимся, что $\Psi_{g_{2}}$ не содержит в качестве факторов квадратов с периодом $2<p \leqslant 16$ или квадратов с одной ошибкой с таким периодом $p$, что $4<p \leqslant 16$. Заметим, что длина фактор-прообразов таких факторов не превосходит 7. Поэтому нам достаточно проверить, что в $\Omega$ фактор-образ любого фактора длины 7 при отображении $f_{g_{2}}$ не содержит таких факторов. Согласно лемме 1 любой фактор длины 7 в $\Omega$ является одним из 22 слов, перечисленных в утверждении леммы. Таким образом, используя компьютерные вычисления, достаточно проверить, что при отображении $f_{g_{2}}$ образ любого из этих 22 слов не содержит таких квадратов или квадратов с одной ошибкой.

Теперь докажем, что $\Psi_{g_{2}}$ не содержит факторов, являющихся квадратами с периодом больше 16. Предположим обратное: пусть $\Psi_{g_{2}}$ содержит квадрат $w$ с периодом, большим 16. Заметим, что в $\Psi_{g_{2}}$ любой фактор длины 16 содержит в качестве фактора монообраз, поэтому левый корень этого квадрата содержит некоторый фактор $\Psi_{g_{2}}[i, i+5]$, являющийся монообразом. Отметим, что $\Psi_{g_{2}}[i, i+5]=\Psi_{g_{2}}[i+p, i+p+5]$. Предположим, что $\Psi_{g_{2}}[i+p, i+p+5]$ не является монообразом. Тогда $\Psi_{g_{2}}[i+p, i+p+5]$ содержится в качестве строгого фактора в конкатенации двух последовательных монообразов в $\Psi_{g_{2}}$. Тем самым получаем противоречие с тем, что, как несложно проверить, ни один элемент отображения $g_{2}$ не является строгим фактором конкатенации двух последовательных монообразов этого отображения. Таким образом, $\Psi_{g_{2}}[i+p, i+p+5]$ является монообразом. Следовательно, период квадрата $w$ кратен длине отображения $g$, т.е. $w \sim u_{1} \gamma_{1} \ldots \gamma_{k} u_{2} \mid v_{1} \delta_{1} \ldots \delta_{k} v_{2}$, где $k \geqslant 2$ и $\gamma_{1}, \ldots, \gamma_{k}, \delta_{1}, \ldots, \delta_{k}$ - такие монообразы, что $\gamma_{i}=\delta_{i}, i=1,2, \ldots, k$, и $0 \leqslant\left|u_{1}\right|,\left|u_{2}\right|<6, u_{1}=v_{1}, u_{2}=v_{2}$.

Пусть $\left|u_{1}\right|=0$, т.е. $w \sim \gamma_{1} \ldots \gamma_{k} \mid \delta_{1} \ldots \delta_{k}$. Тогда, поскольку все элементы $g_{2}$ различны, квадрат $w$ является уникальным, что противоречит лемме 2.

Теперь рассмотрим случай $\left|u_{1}\right|>0$, т.е. $w \sim u_{1} \gamma_{1} \ldots \gamma_{k} u_{2} \mid v_{1} \delta_{1} \ldots \delta_{k} v_{2}$, где $0<$ $\left|u_{1}\right|,\left|u_{2}\right|<6$. Заметим, что в этом случае $u_{2} v_{1}$ является монообразом, тем самым $\left|u_{2}\right|+\left|v_{1}\right|=\left|u_{2}\right|+\left|u_{1}\right|=6$. Рассмотрим произвольный прообраз $q$ квадрата $w$. Заметим, что $q$ имеет длину $2 k+4$, при этом $\gamma_{i}=g_{2}(q[i+1] q[i+2])$, $\delta_{i}=g_{2}(q[i+k+2] q[i+k+3])$ для $i=1,2, \ldots, k$. Таким образом, поскольку все элементы $g_{2}$ различны, буквы $q[2], q[3], \ldots, q[2 k+3]$ прообраза $q$ однозначно определяются квадратом $w$. Отметим, что в силу того, что $\gamma_{i}=\delta_{i}$ для $i=1,2, \ldots, k$, то $q[i+1] q[i+2]=q[i+k+2][i+k+3]$ для $i=1,2, \ldots, k$. Следовательно, $q[i]=q[i+k+1]$ для $i=2,3, \ldots, k+2$. Заметим также, что $u_{1}$ является суффиксом в $g_{2}(q[1] q[2])$, а $v_{2}$ является префиксом в $g_{2}(q[2 k+3] q[2 k+4])$. Рассмотрим отдельно следующие подслучаи.

(1) Пусть $\left|u_{1}\right| \geqslant 2$. Заметим, что все элементы $g_{2}$ имеют различные суффиксы длины 2 , кроме элементов, $g_{2}(00)$ и $g_{2}(11)$. Следовательно, элемент $g_{2}(\alpha \beta)$ однозначно определяется своим суффиксом длины 2 и буквой $\beta$. Поэтому из того, что $q[2]=q[k+3]$ следует, что $q[1]=q[k+2]$. Следовательно, $q[1] \ldots q[k+1]=q[k+2] \ldots q[2 k+2]$ и $q[k+2]=q[2 k+3]$, что противоречит сильной бескубности $\Omega$.

(2) Пусть $\left|u_{1}\right|=1$. Следовательно, $\left|v_{2}\right|=5$. Заметим, что все элементы $g_{2}$ имеют различные префиксы длины 5. Поэтому из того, что $u_{2}=v_{2}$, следует, что $q[k+2][k+3]=q[2 k+3] q[2 k+4]$. Следовательно, $q[2] \ldots q[k+2]=q[k+3] \ldots q[2 k+3]$ и $q[k+3]=q[2 k+4]$, что противоречит сильной бескубности $\Omega$.

Таким образом, для $\Psi_{g_{2}}$ мы доказали свойство $\left(\begin{array}{l}* \\ 2\end{array}\right)$. 
Теперь докажем, что $\Psi_{g_{2}}$ не содержит факторов, являющихся квадратами с одной ошибкой и периодом, большим 16. Предположим, что $\Psi_{g_{2}}$ содержит квадрат с одной ошибкой и с периодом $p$, большим 16 . Заметим, что в $\Psi_{g_{2}}$ любой фактор длины больше 16 содержит в качестве факторов 2 монообраза, поэтому левый корень этого квадрата содержит некоторые факторы $\Psi_{g_{2}}[i, i+5]$ и $\Psi_{g_{2}}[i+6, i+11]$, являющихся монообразами. Следовательно, $\Psi_{g_{2}}[i+p, i+p+5]$ и $\Psi_{g_{2}}[i+p+6, i+p+11]$ являются монообразами (аналогично доказательству теоремы 1). Следовательно, период квадрата $w$ кратен длине отображения $g$, т.е. $w \sim u_{1} \gamma_{1} \ldots \gamma_{k} u_{2} \mid v_{1} \delta_{1} \ldots \delta_{k} v_{2}$, где $k \geqslant 2$ и $\gamma_{1}, \ldots, \gamma_{k}, \delta_{1}, \ldots, \delta_{k}-$ монообразы, и $0 \leqslant\left|u_{1}\right|,\left|u_{2}\right|<6,\left|u_{1}\right|=\left|v_{1}\right|,\left|u_{2}\right|=\left|v_{2}\right|$. Несложно проверить, что любые 2 элемента отображения $g_{2}$ либо совпадают, либо отличаются более чем одной буквой. Следовательно, $\gamma_{i}=\delta_{i}, i=1,2, \ldots, k$, поскольку корни квадрата $w$ отличаются одной буквой. Поэтому $\left|u_{1}\right|>0$ и либо $u_{1}$ отличается одной буквой от $v_{1}$, либо $u_{2}$ отличается одной буквой от $v_{2}$. Заметим, что $u_{2} v_{1}$ является монообразом, тем самым $\left|u_{2}\right|+\left|v_{1}\right|=\left|u_{1}\right|+\left|v_{2}\right|=6$. Рассмотрим произвольный прообраз $q$ квадрата $w$. Заметим, что $q$ имеет длину $2 k+4$, при этом $\gamma_{i}=g_{2}(q[i+1] q[i+2]), \delta_{i}=g_{2}(q[i+k+2] q[i+k+3])$ для $i=1,2, \ldots, k$. Таким образом, поскольку все элементы $g_{2}$ различны, буквы $q[2], q[3], \ldots, q[2 k+3]$ прообраза $q$ однозначно определяются квадратом $w$ и $q[2, k+2]=q[k+3,2 k+3]$. Заметим также, что $u_{1}$ является суффиксом в $g_{2}(q[1] q[2])$, а $v_{2}$ является префиксом в $g_{2}(q[2 k+3] q[2 k+4])$. Рассмотрим отдельно следующие подслучаи.

(1) Пусть $\left|u_{1}\right| \geqslant 3$. Заметим, что $\gamma_{1}=\delta_{1}$, поэтому $q[2]=q[k+3]$. Отметим, что любые два суффикса различных элементов $g_{2}(0 \beta)$ и $g_{2}(1 \beta)$, где $\beta \in\{0,1\}$, длины больше 2 отличаются, как минимум, двумя буквами. Следовательно, $u_{1}=v_{1}$. Так как $u_{1}=v_{1}$, то $q[1] q[2]=q[k+2][k+3]$, то есть $q[1]=q[k+2]=$ $q[2 k+3]$, что противоречит сильной бескубности $\Omega$.

(2) Пусть $\left|u_{2}\right| \geqslant 4$ и $u_{2}=v_{2}$. Отметим, что любые два префикса длины большей либо равной 4 различных элементов отображения $g_{2}$ не совпадают. Поэтому $q[k+2] q[k+3]=q[2 k+3] q[2 k+4]$, то есть $q[2]=q[k+3]=q[2 k+4]$, что противоречит сильной бескубности $\Omega$.

(3) Пусть $\left|u_{2}\right| \geqslant 4$ и $u_{2} \neq v_{2}$, то есть $u_{2}$ и $v_{2}$ отличаются одной буквой. Отметим, что $q[2]=q[k+3]$ и $q[k+2]=q[2 k+3]$. Поэтому $u_{2}$ и $v_{2}$ являются префиксами различных элементов $g_{2}(\alpha 0)$ и $g_{2}(\alpha 1)$, где $\alpha \in\{0,1\}$ имеющих длину больше 3 и отличающимися одной буквой. Это возможно лишь в случае, когда либо $u_{2}=0111, v_{2}=0110$, либо $u_{2}=0110, v_{2}=0111$. Пусть $u_{2}=0111, v_{2}=$ 0110. Тогда $u_{1}=v_{1}=11$, следовательно, $q[1]=q[k+2]=q[2 k+3]$, что противоречит сильной бескубности $\Omega$. Пусть $u_{2}=0110, v_{2}=0111$. Тогда $u_{1}=v_{1}=01$, следовательно, $q[1]=q[k+2]=q[2 k+3]$, что противоречит сильной бескубности $\Omega$.

Таким образом, для $\Psi_{g_{2}}$ мы доказали свойство $\left(\begin{array}{c}* * \\ 4\end{array}\right)$. Теорема доказана.

Из теорем 1 и 2 в силу утверждения 1 можно сделать следующий вывод.

Следствие 1. Если $k_{0}=2, k_{1} \geqslant 4$ или $k_{0} \geqslant 3, k_{1} \geqslant 3$, то существует сверхслово над алфавитом из двух букв, обладающее свойствами $\left(\begin{array}{c}* \\ k_{0}\end{array}\right)$ u $\left(\begin{array}{l}* * \\ k_{1}\end{array}\right)$.

С другой стороны, используя стандартную процедуру компьютерного поиска в глубину в дереве всех слов над алфавитом из двух букв, можно доказать следующие утверждения:

Теорема 3. Не существует сверхслова над алфавитом из двух букв, обладающего свойствами $\left(\begin{array}{c}* \\ 2\end{array}\right)$ u $\left(\begin{array}{c}* * \\ 3\end{array}\right)$. 
Теорема 4. Не существует сверхслова над алфавитом из двух букв, обладающего свойством $\left(\begin{array}{l}* \\ 1\end{array}\right)$.

Из теоремы 4 и утверждения 2 вытекает, что не существует сверхслова над алфавитом из двух букв, обладающего свойством $\left(\begin{array}{l}* \\ 0\end{array}\right)$.

Из теоремы 3 и утверждения 2 вытекает, что не существует сверхслова над алфавитом из двух букв, обладающего свойствами $\left(\begin{array}{c}* \\ 2\end{array}\right)$ и $\left(\begin{array}{c}* * \\ 2\end{array}\right)$.

Таким образом, получаем следующий критерий.

Теорема 5. Над алфавитом из двух букв существует сверхслово, обладающее свойствами $\left(\begin{array}{c}* \\ k_{0}\end{array}\right)$ u $\left(\begin{array}{c}* * \\ k_{1}\end{array}\right)$, тогда и только тогда, когда $k_{0}=2, k_{1} \geqslant 4$ или $k_{0} \geqslant 3$, $k_{1} \geqslant 3$.

Полученные результаты можно наглядно представить в следующей таблице. В данной табличке в каждой ячейке, соответствующей паре $k_{1}, k_{0}, k_{1} \geqslant k_{0}$, указана максимальная длина слова над двухбуквенным алфавитом, обладающего свойствами $\left(\begin{array}{c}* \\ k_{0}\end{array}\right)$ и $\left(\begin{array}{c}* * \\ k_{1}\end{array}\right)$ (в случае, если существует сверхслово, обладающее данными свойствами, указано значение $\infty$; черными квадратами отмечены клетки с $\left.k_{1}<k_{0}\right)$.

Таблица 1

\begin{tabular}{|c|c|c|c|c|c|}
\hline$k_{1} \backslash k_{0}$ & 0 & 1 & 2 & 3 & $\ldots$ \\
\hline 1 & 3 & 6 & $\mathbf{\square}$ & $\mathbf{\square}$ & $\ldots$ \\
\hline 2 & 3 & 11 & 16 & $\mathbf{\square}$ & $\ldots$ \\
\hline 3 & 3 & 11 & 152 & $\infty$ & $\ldots$ \\
\hline 4 & 3 & 12 & $\infty$ & $\infty$ & $\ldots$ \\
\hline 5 & 3 & 15 & $\infty$ & $\infty$ & $\ldots$ \\
\hline 6 & 3 & 18 & $\infty$ & $\infty$ & $\ldots$ \\
\hline$\ldots$ & $\ldots$ & $\ldots$ & $\ldots$ & $\ldots$ & $\ldots$ \\
\hline
\end{tabular}

\section{5. Трехбуквенный алфавит}

Теорема 6. Существует сверхслово над алфавитом из трех букв, которое обладает свойствами $\left(\begin{array}{c}* \\ 0\end{array}\right)$ u $\left(\begin{array}{c}* * \\ 4\end{array}\right)$.

Доказательство. Зададим $g_{3}: 00 \mapsto 012021,01 \mapsto 012102,10 \mapsto 120102,11 \mapsto$ 120210. Покажем, что сверхслово $\Psi_{g_{3}}$ обладает свойствами $\left(\begin{array}{l}* \\ 0\end{array}\right)$ и $\left(\begin{array}{c}* * \\ 4\end{array}\right)$.

Сначала убедимся, что $\Psi_{g_{3}}$ не содержит в качестве факторов квадратов с периодом $p_{1}$ или квадратов с одной ошибкой и с таким периодом $p_{2}$, что $p_{1} \leqslant 16$, $4<p_{2} \leqslant 16$. Заметим, что длина фактор-прообразов таких факторов не превосходит 7. Поэтому нам достаточно проверить, что фактор-образ любого фактора $\Omega$ длины 7 при отображении $f_{g_{3}}$ не содержит таких факторов. Согласно лемме 1 любой фактор длины 7 в $\Omega$ является одним из 22 слов, перечисленных в утверждении леммы. Таким образом, используя компьютерные вычисления, достаточно проверить, что при отображении $f_{g_{3}}$ образ любого из этих 22 слов не содержит таких квадратов или квадратов с одной ошибкой.

Теперь докажем, что $\Psi_{g_{3}}$ не содержит факторов, являющихся квадратами с периодом больше 16. Предположим обратное: пусть $\Psi_{g_{3}}$ содержит квадрат $w$ с периодом, большим 16. Заметим, что в $\Psi_{g_{3}}$ любой фактор длины 16 содержит в качестве фактора монообраз, поэтому левый корень этого квадрата содержит некоторый фактор $\Psi_{g_{3}}[i, i+5]$, являющийся монообразом. Отметим, что $\Psi_{g_{3}}[i, i+5]=\Psi_{g_{3}}[i+p, i+p+5]$. Предположим, что $\Psi_{g_{3}}[i+p, i+p+5]$ не является монообразом. Тогда $\Psi_{g_{3}}[i+p, i+p+5]$

3 Дискретная математика, т. 27 № 2 
содержится в качестве строгого фактора в конкатенации двух последовательных монообразов в $\Psi_{g_{3}}$. Тем самым получаем противоречие с тем, что, как несложно проверить, ни один элемент отображения $g_{3}$ не является строгим фактором конкатенации двух последовательных монообразов этого отображения. Таким образом, $\Psi_{g_{3}}[i+p, i+p+5]$ является монообразом. Следовательно, период квадрата $w$ кратен длине отображения $g$, т.е. $w \sim u_{1} \gamma_{1} \ldots \gamma_{k} u_{2} \mid v_{1} \delta_{1} \ldots \delta_{k} v_{2}$, где $k \geqslant 2$ и $\gamma_{1}, \ldots, \gamma_{k}, \delta_{1}, \ldots, \delta_{k}$ - такие монообразы, что $\gamma_{i}=\delta_{i}, i=1,2, \ldots, k$, и $0 \leqslant\left|u_{1}\right|,\left|u_{2}\right|<6, u_{1}=v_{1}, u_{2}=v_{2}$.

Пусть $\left|u_{1}\right|=0$, т.е. $w \sim \gamma_{1} \ldots \gamma_{k} \mid \delta_{1} \ldots \delta_{k}$. Тогда, поскольку все элементы $g_{3}$ различны, квадрат $w$ является уникальным, что противоречит лемме 2.

Теперь рассмотрим случай $\left|u_{1}\right|>0$, т.е. $w \sim u_{1} \gamma_{1} \ldots \gamma_{k} u_{2} \mid v_{1} \delta_{1} \ldots \delta_{k} v_{2}$, где $0<$ $\left|u_{1}\right|,\left|u_{2}\right|<6$. Заметим, что в этом случае $u_{2} v_{1}$ является монообразом, тем самым $\left|u_{2}\right|+\left|v_{1}\right|=\left|u_{2}\right|+\left|u_{1}\right|=6$. Рассмотрим произвольный прообраз $q$ квадрата $w$. Заметим, что $q$ имеет длину $2 k+4$, при этом $\gamma_{i}=g_{3}(q[i+1] q[i+2])$, $\delta_{i}=g_{3}(q[i+k+2] q[i+k+3])$ для $i=1,2, \ldots, k$. Таким образом, поскольку все элементы $g_{3}$ различны, буквы $q[2], q[3], \ldots, q[2 k+3]$ прообраза $q$ однозначно определяются квадратом $w$. Отметим, что $q[i+1] q[i+2]=q[i+k+2][i+k+3]$ для $i=1,2, \ldots, k$ в силу того, что $\gamma_{i}=\delta_{i}$ для $i=1,2, \ldots, k$, . Следовательно, $q[i]=q[i+k+1]$ для $i=2,3, \ldots, k+2$. Заметим также, что $u_{1}$ является суффиксом в $g_{3}(q[1] q[2])$, а $v_{2}$ является префиксом в $g_{3}(q[2 k+3] q[2 k+4])$. Рассмотрим отдельно следующие подслучаи.

(1) Пусть $\left|u_{1}\right| \geqslant 2$. Заметим, что все элементы $g_{3}$ имеют различные суффиксы длины 2 , кроме элементов $g_{3}(10)$ и $g_{3}(01)$. Поэтому из равенства $q[2]=q[k+3]$ следует $q[1]=q[k+2]$. Значит, $q[1] \ldots q[k+1]=q[k+2] \ldots q[2 k+2]$ и $q[k+2]=$ $q[2 k+3]$, что противоречит сильной бескубности $\Omega$.

(2) Пусть $\left|u_{1}\right|=1$. Следовательно, $\left|v_{2}\right|=5$. Заметим, что все элементы $g_{3}$ имеют различные префиксы длины 5 . Поэтому из $u_{2}=v_{2}$ следует $q[k+2][k+3]=$ $q[2 k+3] q[2 k+4]$. Значит, $q[2] \ldots q[k+2]=q[k+3] \ldots q[2 k+3]$ и $q[k+3]=q[2 k+4]$, что противоречит сильной бескубности $\Omega$.

Таким образом, для $\Psi_{g_{3}}$ мы доказали свойство $\left(\begin{array}{l}* \\ 0\end{array}\right)$.

Теперь докажем, что $\Psi_{g_{3}}$ не содержит факторов, являющихся квадратами с одной ошибкой и периодом, большим 16. Предположим, что $\Psi_{g_{3}}$ содержит квадрат с одной ошибкой и с периодом $p$, большим 16. Заметим, что в $\Psi_{g_{3}}$ любой фактор длины больше 16 содержит в качестве фактора 2 монообраза, поэтому левый корень этого квадрата содержит некоторые факторы $\Psi_{g_{3}}[i, i+5]$ и $\Psi_{g_{3}}[i+6, i+11]$, являющихся монообразами. Аналогично доказательству теоремы 1.1 получаем, что $\Psi_{g_{3}}[i+p, i+$ $p+5]$ и $\Psi_{g_{3}}[i+p+6, i+p+11]$ являются монообразами. Следовательно, период квадрата $w$ кратен длине отображения $g_{3}$, т.е. $w \sim u_{1} \gamma_{1} \ldots \gamma_{k} u_{2} \mid v_{1} \delta_{1} \ldots \delta_{k} v_{2}$, где $k \geqslant 2$ и $\gamma_{1}, \ldots, \gamma_{k}, \delta_{1}, \ldots, \delta_{k}-$ монообразы, и $0 \leqslant\left|u_{1}\right|,\left|u_{2}\right|<6,\left|u_{1}\right|=\left|v_{1}\right|,\left|u_{2}\right|=\left|v_{2}\right|$.

Пусть $\left|u_{1}\right|=0$, то есть $w$ имеет вид $\gamma_{1} \ldots \gamma_{k} \mid \delta_{1} \ldots \delta_{k}$, где $\gamma_{1}, \ldots, \gamma_{k}, \delta_{1}, \ldots, \delta_{k}$ являются монообразами. Следовательно, $w$ содержит 2 монообраза $\gamma_{i}$ и $\delta_{i}$, которые отличаются друг от друга ровно одной буквой. Несложно проверить, что любые 2 элемента отображения $g_{3}$ либо совпадают, либо отличаются более чем одной буквой. Следовательно, такой случай невозможен.

Теперь рассмотрим случай $\left|u_{1}\right|>0$, т.е. $w \sim u_{1} \gamma_{1} \ldots \gamma_{k} u_{2} \mid v_{1} \delta_{1} \ldots \delta_{k} v_{2}$, где $0<$ $\left|u_{1}\right|,\left|u_{2}\right|<6$. Заметим, что в этом случае $u_{2} v_{1}$ является монообразом, тем самым $\left|u_{2}\right|+\left|v_{1}\right|=\left|u_{1}\right|+\left|v_{2}\right|=6$. Рассмотрим произвольный прообраз $q$ квадрата $w$. Заметим, что $q$ имеет длину $2 k+4$, при этом $\gamma_{i}=g_{3}(q[i+1] q[i+2])$, $\delta_{i}=g_{3}(q[i+k+2] q[i+k+3])$ для $i=1,2, \ldots, k$. Таким образом, поскольку все 
элементы $g_{3}$ различны, буквы $q[2], q[3], \ldots, q[2 k+3]$ прообраза $q$ однозначно определяются квадратом $w$. Заметим также, что $u_{1}$ является суффиксом в $g_{3}(q[1] q[2])$, а $v_{2}$ является префиксом в $g_{3}(q[2 k+3] q[2 k+4])$. Так как любые 2 элемента отображения $g_{3}$ отличаются, по крайней мере, на 2 буквы, то $\gamma_{i}=\delta_{i}, i=1, \ldots, k$. Следовательно, $q[2, k+2]=q[k+3,2 k+3]$ и либо $u_{1}$ отличается на одну букву от $v_{1}$, либо $u_{2}$ отличается на одну букву от $v_{2}$. Рассмотрим отдельно следующие подслучаи.

(1) Пусть $\left|u_{1}\right| \geqslant 2$. Отметим, что $\gamma_{1}=\delta_{1}$, поэтому $q[2]=q[k+3]$. Отметим, что любые два суффикса длины больше 1 различных элементов $g_{3}(0 \alpha)$ и $g_{3}(1 \alpha)$, где $\alpha \in 0,1$ различаются, как минимум, по двум буквам. Поэтому в этом случае, $u_{1}=v_{1}$. Следовательно, $q[1] q[2]=q[k+2] q[k+3]$, значит $q[1]=$ $q[k+2]=q[2 k+3]$, что противоречит сильной бескубности $\Omega$.

(2) Пусть $\left|u_{1}\right|=1$. Тогда $\left|u_{2}\right|=5$. Отметим, что любые два префикса длины 5 различных элементов $g_{3}$, различаются, как минимум, по двум буквам. Поэтому $u_{2}=v_{2}$. Следовательно, $q[k+2] q[k+3]=q[2 k+3] q[2 k+4]$, откуда $q[2]=q[k+3]=q[2 k+4]$, что противоречит сильной бескубности $\Omega$.

Таким образом, для $\Psi_{g_{3}}$ мы доказали свойство $\left(\begin{array}{c}* * \\ 4\end{array}\right)$. Теорема доказана.

Теорема 7. Существует бесконечное слово над алфавитом из трех букв, которое обладает свойствами $\left(\begin{array}{c}* \\ 1\end{array}\right)$ u $\left(\begin{array}{c}* * \\ 1\end{array}\right)$.

Доказательство. Зададим $g_{4}: 00 \mapsto 00,01 \mapsto 11,10 \mapsto 22,11 \mapsto 00$. Покажем, что для сверхслова $\Psi_{g_{4}}$ выполняются свойства $\left(\begin{array}{c}* \\ 1\end{array}\right)$ и $\left(\begin{array}{c}* * \\ 1\end{array}\right)$.

Сначала убедимся, что $\Psi_{g_{4}}$ не содержит в качестве факторов квадратов с периодом $p_{1}$ или квадратов с одной ошибкой с периодом $p_{2}$ таких, что $1<p_{1} \leqslant 4$, $1<p_{2} \leqslant 4$. Заметим, что длина фактор-прообразов таких факторов не превосходит 7. Поэтому нам достаточно проверить, что фактор-образ любого фактора $\Omega$ длины 7 при отображении $f_{g_{4}}$ не содержит таких факторов. Согласно лемме 1 любой фактор длины 7 в $\Omega$ является одним из 22 слов, перечисленных в утверждении леммы. Таким образом, используя компьютерные вычисления, достаточно проверить, что при отображении $f_{g_{4}}$ образ любого из этих 22 слов не содержит таких квадратов или квадратов с одной ошибкой.

Теперь докажем, что $\Psi_{g_{4}}$ не содержит факторов, являющихся квадратами с периодом больше 4. Предположим обратное: пусть $\Psi_{g_{4}}$ содержит квадрат $w$ с периодом, большим 4. Заметим, что в $\Psi_{g_{4}}$ любой фактор длины 4 содержит в качестве фактора монообраз, поэтому левый корень этого квадрата содержит некоторый фактор $\Psi_{g_{4}}[i, i+1]$, являющийся монообразом. Отметим, что $\Psi_{g_{4}}[i, i+1]=\Psi_{g_{4}}[i+p, i+p+1]$. Предположим, что $\Psi_{g_{4}}[i+p, i+p+1]$ не является монообразом. Тогда $\Psi_{g_{4}}[i+p, i+p+1]$ содержится в качестве строгого фактора в конкатенации двух последовательных монообразов в $\Psi_{g_{4}}$. Тем самым получаем противоречие с тем, что, как несложно проверить, ни один элемент отображения $g_{4}$ не является строгим фактором конкатенации двух последовательных монообразов этого отображения. Таким образом, $\Psi_{g_{4}}[i+p, i+p+1]$ является монообразом. Следовательно, период квадрата $w$ кратен длине отображения $g_{4}$, т.е. $w \sim u_{1} \gamma_{1} \ldots \gamma_{k} u_{2} \mid v_{1} \delta_{1} \ldots \delta_{k} v_{2}$, где $k \geqslant 2$ и $\gamma_{1}, \ldots, \gamma_{k}, \delta_{1}, \ldots, \delta_{k}$ - такие монообразы, что $\gamma_{i}=\delta_{i}, i=1,2, \ldots, k$, и $0 \leqslant\left|u_{1}\right|,\left|u_{2}\right|<2, u_{1}=v_{1}, u_{2}=v_{2}$.

Пусть $\left|u_{1}\right|=0$, т.е. $w \sim \gamma_{1} \ldots \gamma_{k} \mid \delta_{1} \ldots \delta_{k}$. Несложно заметить, что любой фактор $\Psi_{g_{4}}$, состоящий из двух последовательных монообразов, имеет единственный прообраз. Таким образом, квадрат $w$ является уникальным, что противоречит лемме 2.

Теперь рассмотрим случай $\left|u_{1}\right|>0$, т.е. $w \sim u_{1} \gamma_{1} \ldots \gamma_{k} u_{2} \mid v_{1} \delta_{1} \ldots \delta_{k} v_{2}$, где $k \geqslant 2$ и $\left|u_{1}\right|,\left|u_{2}\right|=1$. Заметим, что в этом случае $u_{2} v_{1}$ является монообразом. Рассмотрим 
произвольный прообраз $q$ квадрата $w$. Заметим, что $q$ имеет длину $2 k+4$, при этом $\gamma_{i}=g_{4}(q[i+1] q[i+2]), \delta_{i}=g_{4}(q[i+k+2] q[i+k+3])$ для $i=1,2, \ldots, k$. Таким образом, поскольку $k \geqslant 2$, факторы $\gamma_{1} \ldots \gamma_{k}$ и $\delta_{1} \ldots \delta_{k}$ имеют единственные прообразы, поэтому буквы $q[2], q[3], \ldots, q[2 k+3]$ прообраза $q$ однозначно определяются квадратом $w$. Отметим, что $q[i+1] q[i+2]=q[i+k+2] q[i+k+3]$ для $i=1,2, \ldots, k$, в силу того, что $k \geqslant 2$ и $\gamma_{i}=\delta_{i}$ для $i=1,2, \ldots, k$. Следовательно, $q[i]=q[i+k+1]$ для $i=2,3, \ldots, k+2$. Заметим также, что $u_{1}$ является суффиксом в $g_{4}(q[1] q[2])$, а $v_{2}$ является префиксом в $g_{4}(q[2 k+3] q[2 k+4])$. Рассмотрим отдельно следующие подслучаи.

(1) Пусть $u_{1}=1$. Тогда $q[2]=q[k+3]=1, q[1]=q[k+2]=0$. Следовательно, $q[1] \ldots q[k+1]=q[k+2] \ldots q[2 k+2]$ и $q[k+2]=q[2 k+3]$, что противоречит сильной бескубности $\Omega$.

(2) Пусть $u_{1}=2$. Тогда $q[2]=q[k+3]=0, q[1]=q[k+2]=1$. Следовательно, $q[1] \ldots q[k+1]=q[k+2] \ldots q[2 k+2]$ и $q[k+2]=q[2 k+3]$, что противоречит сильной бескубности $\Omega$.

(3) Пусть $u_{1}=0$. Тогда $q[1] q[2]$ и $q[k+2] q[k+3]$ равны 00 или 11 , поэтому из $q[2]=$ $q[k+3]$ следует $q[1]=q[k+2]$. Следовательно, $q[1] \ldots q[k+1]=q[k+2] \ldots q[2 k+2]$ и $q[k+2]=q[2 k+3]$, что противоречит сильной бескубности $\Omega$.

Таким образом, для $\Psi_{g_{4}}$ мы доказали свойство $\left(\begin{array}{l}* \\ 1\end{array}\right)$.

Теперь докажем, что $\Psi_{g_{4}}$ не содержит факторов, являющихся квадратами с одной ошибкой и периодом, большим 4. Предположим, что $\Psi_{g_{4}}$ содержит квадрат $w$ с одной ошибкой и с периодом $p$, большим 4. Заметим, что в $\Psi_{g_{4}}$ любой фактор длины больше 4 содержит в качестве фактора 2 монообраза, поэтому левый корень этого квадрата содержит некоторые факторы $\Psi_{g_{4}}[i, i+1]$ и $\Psi_{g_{4}}[i+2, i+3]$, являющихся монообразами. Следовательно, аналогично доказательству теоремы 1.1, $\Psi_{g_{4}}[i+p, i+p+1]$ и $\Psi_{g_{4}}[i+p+2, i+p+3]$ являются монообразами. Значит, период квадрата $w$ кратен длине отображения $g_{4}$, т.е. $w \sim u_{1} \gamma_{1} \ldots \gamma_{k} u_{2} \mid v_{1} \delta_{1} \ldots \delta_{k} v_{2}$, где $k \geqslant 2$ и $\gamma_{1}, \ldots, \gamma_{k}, \delta_{1}, \ldots, \delta_{k}$ - монообразы, $i=1,2, \ldots, k$, и $0 \leqslant\left|u_{1}\right|,\left|u_{2}\right|<2,\left|u_{1}\right|=\left|v_{1}\right|$, $\left|u_{2}\right|=\left|v_{2}\right|$.

Пусть $\left|u_{1}\right|=0$, то есть $w$ имеет вид $\gamma_{1} \ldots \gamma_{k} \mid \delta_{1} \ldots \delta_{k}$, где $\gamma_{1}, \ldots, \gamma_{k}, \delta_{1}, \ldots, \delta_{k}$. Следовательно, $w$ содержит 2 монообраза, которые отличаются друг от друга ровно одной буквой. Несложно проверить, что любые 2 элемента отображения $g_{4}$ либо совпадают, либо отличаются более чем одной буквой. Следовательно, такой случай невозможен.

Теперь рассмотрим случай $\left|u_{1}\right|=1$, т.е. $w \sim u_{1} \gamma_{1} \ldots \gamma_{k} u_{2} \mid v_{1} \delta_{1} \ldots \delta_{k} v_{2}$, где $k \geqslant 2$ и $\left|u_{1}\right|,\left|u_{2}\right|=1$. Заметим, что в этом случае $u_{2} v_{1}$ является монообразом. Рассмотрим произвольный прообраз $q$ квадрата $w$. Заметим, что $q$ имеет длину $2 k+4$, при этом $\gamma_{i}=g_{4}(q[i+1] q[i+2]), \delta_{i}=g_{4}(q[i+k+2] q[i+k+3])$ для $i=1,2, \ldots, k$. Поэтому аналогично доказательству свойства $\left(\begin{array}{l}* \\ 1\end{array}\right)$, буквы $q[2], q[3], \ldots, q[2 k+3]$ прообраза $q$ однозначно определяются квадратом $w$. Поскольку элементы отображения $g_{4}$ не могут отличаться ровно одной буквой, имеем $\gamma_{i}=\delta_{i}$ для $i=1,2, \ldots, k$, поэтому аналогично доказательству свойства $\left(\begin{array}{l}* \\ 1\end{array}\right)$ получаем $q[i+1] q[i+2]=q[i+k+2] q[i+k+3]$ для $i=1,2, \ldots, k$. Заметим также, что $u_{1}$ является суффиксом в $g_{4}(q[1] q[2])$, а $v_{2}$ является префиксом в $g_{4}(q[2 k+3] q[2 k+4])$. Отметим, что либо $u_{1}$ не равно $v_{1}$, либо $u_{2}$ не равно $v_{2}$. Рассмотрим отдельно следующие подслучаи.

(1) Пусть $u_{1}=v_{1}$. Тогда $g_{4}(q[1] q[2])=g_{4}(q[k+2] q[k+3])$, т.е. $q[1] q[2]$ и $q[k+2] q[k+$ $3]$ равны 00 либо 11. Поэтому из $q[2]=q[k+3]$ следует $q[1]=q[k+2]=q[2 k+3]$, что противоречит сильной бескубности $\Omega$. 
(2) Пусть $u_{2}=v_{2}$. Тогда $g_{4}(q[k+2] q[k+3])=g_{4}(q[2 k+3] q[2 k+4])$, т.е. $q[k+2] q[k+3]$ и $q[2 k+3] q[2 k+4]$ равны 00 либо 11. Поэтому из $q[k+2]=q[2 k+3]$ следует $q[2]=q[k+3]=q[2 k+4]$, что противоречит сильной бескубности $\Omega$.

Таким образом, для $\Psi_{g_{4}}$ мы доказали свойство $\left(\begin{array}{c}* * \\ 1\end{array}\right)$. Теорема доказана.

Из теорем 2 и 7 с учетом утверждения 1 можно сделать следующий вывод.

Следствие 2. Если $k_{0}=0, k_{1} \geqslant 4$ или $k_{0} \geqslant 1, k_{1} \geqslant 1$, то существует сверхслово над алфавитом из трех букв, обладающее свойствами $\left(\begin{array}{c}* \\ k_{0}\end{array}\right)$ u $\left(\begin{array}{l}* * \\ k_{1}\end{array}\right)$.

С другой стороны, используя стандартную процедуру компьютерного поиска в глубину в дереве всех слов над алфавитом из трех букв, можно доказать следующее утверждение.

Теорема 8. Не существует сверхслова над алфавитом из трех букв, обладающего свойствами $\left(\begin{array}{c}* \\ 0\end{array}\right)$ u $\left(\begin{array}{c}* * \\ 3\end{array}\right)$.

Из теоремы 8 и утверждения 2 вытекает, что не существует сверхслова над алфавитом из трех букв, обладающего свойствами $\left(\begin{array}{c}* \\ k_{0}\end{array}\right)$ и $\left(\begin{array}{c}* * \\ k_{1}\end{array}\right), k_{0}=0, k_{1} \leqslant 3$.

Таким образом, получаем следующий критерий.

Теорема 9. Над алфавитом из трех букв существует сверхслово, обладающее свойствами $\left(\begin{array}{c}* \\ k_{0}\end{array}\right)$ u $\left(\begin{array}{c}* * \\ k_{1}\end{array}\right)$, тогда и только тогда, когда $k_{0}=0, k_{1} \geqslant 4$ или $k_{0} \geqslant 1$, $k_{1} \geqslant 1$.

Полученные результаты можно наглядно представить в следующей табличке. В этой табличке в каждой ячейке, соответствующей паре $k_{1}, k_{0}, k_{1} \geqslant k_{0}$, указана максимальная длина слова над трехбуквенным алфавитом, обладающего свойствами $\left(\begin{array}{c}* \\ k_{0}\end{array}\right)$ и $\left(\begin{array}{c}* * \\ k_{1}\end{array}\right)$ (в случае, если существует сверхслово, обладающее данными свойствами, указано значение $\infty$; черные квадраты стоят в клетках с $k_{1}<k_{0}$ ).

Таблица 2

\begin{tabular}{|c|c|c|c|c|}
\hline$k_{1} \backslash k_{0}$ & 0 & 1 & 2 & $\ldots$ \\
\hline 1 & 5 & $\infty$ & $\mathbf{\square}$ & $\ldots$ \\
\hline 2 & 11 & $\infty$ & $\infty$ & $\ldots$ \\
\hline 3 & 47 & $\infty$ & $\infty$ & $\ldots$ \\
\hline 4 & $\infty$ & $\infty$ & $\infty$ & $\ldots$ \\
\hline$\ldots$ & $\ldots$ & $\ldots$ & $\ldots$ & $\ldots$ \\
\hline
\end{tabular}

\section{6. Четырехбуквенный алфавит}

Теорема 10. Существует бесконечное слово над алфавитом из четырех букв, которое обладает свойствами $\left(\begin{array}{c}* \\ 0\end{array}\right) u\left(\begin{array}{c}* * \\ 1\end{array}\right)$.

Доказательство. Зададим $g_{5}$ :

$00 \mapsto 0123021301231021302310321023120321$,

$01 \mapsto 0312013210320130213201230213012310$,

$10 \mapsto 2130231032102312031023012031230132$,

$11 \mapsto 2301203123013203120132103201302103$.

Покажем, что сверхслово $\Psi_{g_{5}}$ обладает свойствами $\left(\begin{array}{c}* \\ 0\end{array}\right)$ и $\left(\begin{array}{c}* * \\ 1\end{array}\right)$.

Сначала убедимся, что $\Psi_{g_{5}}$ не содержит в качестве факторов квадраты с периодом $p \leqslant 100$ или квадраты с одной ошибкой и таким периодом $p$, что $1<p \leqslant 100$. Заметим, что длина фактор-прообразов таких факторов не превосходит 7. Поэтому нам достаточно проверить, что в $\Omega$ фактор-образ любого фактора длины 7 при 
отображении $f_{g_{5}}$ не содержит таких факторов. Согласно лемме 1 любой фактор длины 7 в $\Omega$ является одним из 22 слов, перечисленных в утверждении леммы. Таким образом, используя компьютерные вычисления, достаточно проверить, что при отображении $f_{g_{5}}$ образ любого из этих 22 слов не содержит таких квадратов или квадратов с одной ошибкой.

Теперь докажем, что $\Psi_{g_{5}}$ не содержит факторов, являющихся квадратами с периодом больше 100. Предположим обратное: пусть $\Psi_{g_{5}}$ содержит квадрат $w$ с периодом, большим 100. Заметим, что в $\Psi_{g_{5}}$ любой фактор длины 100 содержит в качестве фактора монообраз, поэтому левый корень этого квадрата содержит некоторый фактор $\Psi_{g_{5}}[i, i+33]$, являющийся монообразом. Отметим, что $\Psi_{g_{5}}[i, i+33]=\Psi_{g_{5}}[i+p, i+p+33]$. Предположим, что $\Psi_{g_{5}}[i+p, i+p+33]$ не является монообразом. Тогда $\Psi_{g_{5}}[i+p, i+p+33]$ содержится в качестве строгого фактора в конкатенации двух последовательных монообразов в $\Psi_{g_{5}}$. Тем самым получаем противоречие с тем, что, как несложно проверить, ни один элемент отображения $g_{5}$ не является строгим фактором конкатенации двух последовательных монообразов этого отображения. Таким образом, $\Psi_{g_{5}}[i+p, i+p+33]$ является монообразом. Следовательно, период квадрата $w$ кратен длине отображения $g_{5}$, т.е. $w \sim u_{1} \gamma_{1} \ldots \gamma_{k} u_{2} \mid v_{1} \delta_{1} \ldots \delta_{k} v_{2}$, где $k \geqslant 2$ и $\gamma_{1}, \ldots, \gamma_{k}, \delta_{1}, \ldots, \delta_{k}$ - такие монообразы, что $\gamma_{i}=\delta_{i}, i=1,2, \ldots, k$, и $0 \leqslant\left|u_{1}\right|,\left|u_{2}\right|<34, u_{1}=v_{1}, u_{2}=v_{2}$. Пусть $\left|u_{1}\right|=0$, т.е. $w \sim \gamma_{1} \ldots \gamma_{k} \mid \delta_{1} \ldots \delta_{k}$. Тогда, поскольку все элементы $g_{5}$ различны, квадрат $w$ является уникальным, что противоречит лемме 2.

Теперь рассмотрим случай $\left|u_{1}\right|>0$, т.е. $w \sim u_{1} \gamma_{1} \ldots \gamma_{k} u_{2} \mid v_{1} \delta_{1} \ldots \delta_{k} v_{2}$, где $0<$ $\left|u_{1}\right|,\left|u_{2}\right|<34$. Заметим, что в этом случае $u_{2} v_{1}$ является монообразом, тем самым $\left|u_{2}\right|+\left|v_{1}\right|=\left|u_{2}\right|+\left|u_{1}\right|=34$. Рассмотрим произвольный прообраз $q$ квадрата $w$. Заметим, что $q$ имеет длину $2 k+4$, при этом $\gamma_{i}=g_{5}(q[i+1] q[i+2])$, $\delta_{i}=g_{5}(q[i+k+2] q[i+k+3])$ для $i=1,2, \ldots, k$. Таким образом, поскольку все элементы $g_{5}$ различны, буквы $q[2], q[3], \ldots, q[2 k+3]$ прообраза $q$ однозначно определяются квадратом $w$. Отметим, что $q[i+1] q[i+2]=q[i+k+2] q[i+k+3]$ для $i=1,2, \ldots, k$, в силу того, что $\gamma_{i}=\delta_{i}$ для $i=1,2, \ldots, k$, . Следовательно, $q[i]=q[i+k+1]$ для $i=2,3, \ldots, k+2$. Заметим также, что $u_{1}$ является суффиксом в $g_{5}(q[1] q[2])$. Отметим, что различные элементы отображения $g_{5}$ заканчиваются разными буквами. Поэтому, так как $u_{1}=v_{1}$, то $q[1] q[2]=q[k+2] q[k+3]$. Следовательно, $q[1] \ldots q[k+1]=q[k+2] \ldots q[2 k+2]$ и $q[k+2]=q[2 k+3]$, что противоречит сильной бескубности $\Omega$. Таким образом, для $\Psi_{g_{5}}$ мы доказали свойство $\left(\begin{array}{l}* \\ 0\end{array}\right)$.

Теперь докажем, что $\Psi_{g_{5}}$ не содержит факторов, являющихся квадратами с одной ошибкой и периодом, большим 100 . Предположим, что $\Psi_{g_{5}}$ содержит квадрат $w$ с одной ошибкой и с периодом $p$, большим 100. Заметим, что в $\Psi_{g_{5}}$ любой фактор длины больше 100 содержит в качестве фактора 2 монообраза, поэтому левый корень этого квадрата содержит некоторые факторы $\Psi_{g_{5}}[i, i+33]$ и $\Psi_{g_{5}}[i+34, i+67]$, являющиеся монообразами. Поэтому аналогично доказательству теоремы $1, \Psi_{g_{5}}[i+p, i+p+33]$ и $\Psi_{g_{5}}[i+p+34, i+p+67]$ являются монообразами. Следовательно, период квадрата $w$ кратен длине отображения $g_{5}$, т.е. $w \sim u_{1} \gamma_{1} \ldots \gamma_{k} u_{2} \mid v_{1} \delta_{1} \ldots \delta_{k} v_{2}$, где $k \geqslant 2$ и $\gamma_{1}, \ldots, \gamma_{k}, \delta_{1}, \ldots, \delta_{k}-$ монообразы, $i=1,2, \ldots, k$, и $0 \leqslant\left|u_{1}\right|,\left|u_{2}\right|<34,\left|u_{1}\right|=\left|v_{1}\right|$, $\left|u_{2}\right|=\left|v_{2}\right|$.

Пусть $\left|u_{1}\right|=0$, то есть $w$ имеет вид $\gamma_{1} \ldots \gamma_{k} \mid \delta_{1} \ldots \delta_{k}$, где $\gamma_{1}, \ldots, \gamma_{k}, \delta_{1}, \ldots, \delta_{k}$. Следовательно, $w$ содержит 2 монообраза, которые отличаются друг от друга ровно одной буквой. Несложно проверить, что любые 2 элемента отображения $g_{5}$ либо 
совпадают, либо отличаются более чем одной буквой. Следовательно, такой случай невозможен.

Теперь рассмотрим случай $\left|u_{1}\right|>0$, т.е. $w \sim u_{1} \gamma_{1} \ldots \gamma_{k} u_{2} \mid v_{1} \delta_{1} \ldots \delta_{k} v_{2}$, где $0<$ $\left|u_{1}\right|,\left|u_{2}\right|<34$. Заметим, что в этом случае $u_{2} v_{1}$ является монообразом, тем самым $\left|u_{2}\right|+\left|v_{1}\right|=\left|u_{1}\right|+\left|v_{2}\right|=34$. Рассмотрим произвольный прообраз $q$ квадрата $w$. Заметим, что $q$ имеет длину $2 k+4$, при этом $\gamma_{i}=g_{5}(q[i+1] q[i+2])$, $\delta_{i}=g_{5}(q[i+k+2] q[i+k+3])$ для $i=1,2, \ldots, k$. Таким образом, поскольку все элементы $g_{5}$ различны, буквы $q[2], q[3], \ldots, q[2 k+3]$ прообраза $q$ однозначно определяются квадратом $w$. Кроме того, аналогично доказательству Теоремы 1 , имеем $q[2, k+2]=q[k+3,2 k+3]$. Заметим также, что $u_{1}$ является суффиксом в $g_{5}(q[1] q[2])$, а $v_{2}$ является префиксом в $g_{5}(q[2 k+3] q[2 k+4])$. Отметим, что либо $u_{1}$ отличается одной буквой от $v_{1}$, либо $u_{2}$ отличается одной буквой от $v_{2}$. Рассмотрим отдельно следующие подслучаи.

(1) Пусть $\left|u_{1}\right| \geqslant 2$. Отметим, что любые два суффикса длины больше либо равной 2 различных элементов $g_{5}$ различаются, как минимум, двумя буквами. Значит, в этом случае $u_{1}=v_{1}$. Следовательно, $q[1] q[2]=q[k+2] q[k+3]$, тем самым $q[1]=q[k+2]=q[2 k+3]$, что противоречит сильной бескубности $\Omega$.

(2) Пусть $\left|u_{1}\right|=1$. Тогда $\left|u_{2}\right|=33$. Отметим, что любые два префикса длины 33 различных элементов $g_{5}$, различаются, как минимум, двумя буквами. Таким образом, $u_{2}=v_{2}$. Поэтому $q[k+2] q[k+3]=q[2 k+3] q[2 k+4]$, следовательно, $q[2]=q[k+3]=q[2 k+4]$, что противоречит сильной бескубности $\Omega$.

Таким образом, для $\Psi_{g_{5}}$ мы доказали свойство $\left(\begin{array}{c}* * \\ 1\end{array}\right)$. Теорема доказана.

Пользуясь теоремами 5,9 и 10 , можно найти $\sigma\left(k_{0}, k_{1}\right)$ для любых $k_{0}, k_{1}$ при $k_{1} \geqslant k_{0}$.

Теорема 11. Справедливы следующие утверждения:

1) если $k_{0}=0$ u $k_{1}<4$, то $\sigma\left(k_{0}, k_{1}\right)=4$,

2) если $k_{0}=0$ и $k_{1}>3$ или $k_{0}=1$ u $k_{1}$ - любое или $k_{0}=2 u k_{1}<4$, то $\sigma\left(k_{0}, k_{1}\right)=3$

3) если $k_{0}=2$ и $k_{1}>3$ или $k_{0}>2 u k_{1}$-любое, то $\sigma\left(k_{0}, k_{1}\right)=2$.

Полученные результаты можно наглядно представить в следующей табличке. В этой табличке в каждой ячейке, соответствующей паре $k_{1}, k_{0}, k_{1} \geqslant k_{0}$, указан минимальный размер алфавита, над которым можно построить сверхслово, обладающее одновременно свойствами $\left(\begin{array}{c}* \\ k_{0}\end{array}\right)$ и $\left(\begin{array}{c}* * \\ k_{1}\end{array}\right)$.

Таблица 3

\begin{tabular}{|c|c|c|c|c|c|}
\hline$k_{1} \backslash k_{0}$ & 0 & 1 & 2 & 3 & $\ldots$ \\
\hline 1 & 4 & 3 & $\mathbf{\square}$ & $\mathbf{\square}$ & $\ldots$ \\
\hline 2 & 4 & 3 & 3 & $\mathbf{\square}$ & $\ldots$ \\
\hline 3 & 4 & 3 & 3 & 2 & $\ldots$ \\
\hline 4 & 3 & 3 & 2 & 2 & $\ldots$ \\
\hline$\ldots$ & $\ldots$ & $\ldots$ & $\ldots$ & $\ldots$ & $\ldots$ \\
\hline
\end{tabular}

\section{Список литературы}

1. Thue A., "Uber unendliche Zeichenreihen", Norske, Vid. Selsk. Skr. I, Mat. Nat. Kl. Khristiana, 7 (1906), 1-22.

2. Саломаа А., Жемчужины теории формальных языков, Пер. с англ., М.: Мир, 1986. 
3. Thue A., "Uber die gegenseitige Lage gleicher Teile gewisser Zeichenreihen", Norske, Vid. Selsk. Skr. I, Mat. Nat. Kl. Kristiania, 1 (1912), 1-67.

4. Fraenkel A. S., Simpson R. J., "How many squares must a binary sequence contain?", Electr. J. Comb., 2 (1995).

5. Crochemore M. , Ilie L., Rytter W., "Repetitions in strings: algorithms and combinatorics", Theor. Comput. Sci., 410:50 (2009), 5227 - 5235.

6. Crochemore M., Rytter W., "Squares, cubes, and time-space efficient string searching", Algorithmica, 13:5 (1995), 405-425.

Статья поступила 17.12.2014. 